\title{
Left upper lobectomy can be a risk factor for thrombosis in the pulmonary vein stump
}

\author{
Kazuto Ohtaka ${ }^{1 *}$, Yasuhiro Hida², Kichizo Kaga ${ }^{2}$, Yasuhiro Takahashi', Hiroshi Kawase', Satoshi Hayama', \\ Tatsunosuke Ichimura', Naoto Senmaru', Naotake Honma² and Yoshiro Matsui ${ }^{2}$
}

\begin{abstract}
Background: Thrombosis in the left upper pulmonary vein stump after left upper lobectomy is a very rare but important complication because it occurs in the systemic circulation system. We previously made the first ever report on the frequency and risk factors of thrombosis in the pulmonary vein stump after lobectomy. In this study, we conducted an investigation in a different hospital to determine whether this was a common complication.

Methods: From 2008 to 2012, 151 patients who underwent lobectomy and following enhanced CT within 2 years after the operation were studied. Postoperative contrast-enhanced CT imaging was retrospectively checked.

Results: We found thrombosis in the pulmonary vein stump in 5 of the 151 patients (3.3\%). All 5 patients underwent left upper lobectomy (17.9\% of the patients who underwent left upper lobectomy). These 5 patients did not have infarction of any vital organ. The thrombus was disappeared several months later on contrast-enhanced $\mathrm{CT}$ in 3 patients and followed in 2 patients. On univariate analysis, there was a significant difference only in the operative procedure $(p<0.001)$.

Conclusions: Thrombosis in the pulmonary vein stump occurred with high frequency in patients who underwent left upper lobectomy. Because the frequency of thrombosis in this study was the same as in our previous report, this might be a common complication.
\end{abstract}

Keywords: Left upper lobectomy, Pulmonary vein stump, Thrombosis, Cerebral infarction

\section{Background}

We previously experienced a case of cerebral infarction with thrombosis in the pulmonary vein (PV) stump that was demonstrated on enhanced CT after left upper lobectomy (LUL). Thereafter, we experienced two other cases with thrombosis in the PV stump after lobectomy without cerebral infarction, and reported these 3 cases [1]. There have been reported 3 cases of thrombosis in the PV stump on chest CT after lobectomy that developed infarction of vital organs as well as 3 other cases with infarction of vital organs after lobectomy but no thrombosis on chest CT [2-6]. All 6 of those patients and our 3 patients underwent LUL. Therefore, we hypothesized that LUL was a risk factor for thrombosis in the PV stump, which could cause infarction of vital organs after lobectomy.

\footnotetext{
*Correspondence: kit_katton0803@yahoo.co.jp

1Department of Thoracic Surgery, Steel Memorial Muroran Hospital, 1-45 Chiribetsu-cho, Muroran, Hokkaido 050-0076, Japan

Full list of author information is available at the end of the article
}

We previously reported a retrospective study that checked the PV stump on postoperative enhanced CT in 193 non-small cell lung cancer (NSCLC) patients who underwent lobectomy [7]. In that study, thrombosis in the PV stump after lobectomy was detected in 7 patients (3.6\%), all of whom underwent LUL. Among the patients who underwent LUL, thrombosis was detected in $13.5 \%$. One of the 7 patients developed cerebral infarction. Our previous report was the first study on thrombosis in the PV stump after lobectomy and clarified that this occurred with a high frequency after LUL.

To determine whether this was a common complication, we conducted the present study on thrombosis in the PV stump after lobectomy in another hospital.

\section{Methods}

The Muroran Steel Memorial Hospital Institutional Review Board approved this retrospective study and waived the requirement for informed concent. 


\section{Patients}

Of the 203 consecutive patients who underwent lobectomy from April 2008 to December 2012 in Muroran Steel Memorial Hospital, 151 patients who underwent contrastenhanced chest-CT at least once within 2 years after the lobectomy were selected. Contrast-enhanced chest CT images for postoperative follow-up in these patients were retrospectively interpreted by a general thoracic surgeon to check for thrombosis in the PV stump. The clinicopathological data were obtained from the medical charts. The pathological staging was based on the 7th edition of the Union Internationale Contra le Cancer (UICC) staging system on tumor-node-metastasis (TNM) classification.

\section{Operative procedure}

Video-assisted thoracoscopic surgery (VATS) or hybrid VATS was performed for all cases except those with large tumor size, chest wall invasion, bronchial invasion, or pulmonary vessel invasion.

Lobectomy was performed as follows. First, the PV was divided using a linear stapler. If the PV could not be divided at that time, one or two branches of it were divided with ligation and the rest of the PV was divided using a linear stapler. Second, a few branches of the pulmonary artery (PA) were divided either by ligation or with a linear stapler. Finally, the bronchus was divided using a linear stapler.

\section{Postoperative adjuvant chemotherapy}

Adjuvant chemotherapy was indicated for all patients who gave informed consent except for the patients with Stage IA. Some of the patients with pT1bN0M0 stage IA took UFT orally for 2 years postoperatively. Other patients underwent intravenous chemotherapy. The patients who discontinued UFT within 2 months and those who discontinued intravenous chemotherapy within 1 cycle were classified into the group without chemotherapy for analysis.

\section{Statistical analysis}

Univariate analyses were performed using the MannWhitney U test or Fisher's exact test. All statistic analyses were performed using Stat Flex ver. 6.0 software (Artech Co., Ltd., Japan). A p value of less than 0.05 was regarded as statistically significant.

\section{Results}

The 151 patients consisted of 101 males and 50 females with a median age of 72 years (range: 38 to 82 years old). Past histories consisted of 79 cases of hypertension, 39 malignant diseases, 22 cases of diabetes mellitus, 19 ischemic heart diseases, 15 arrhythmias, and 15 cerebral vascular diseases. Antithrombotic drugs were taken by 38 patients.

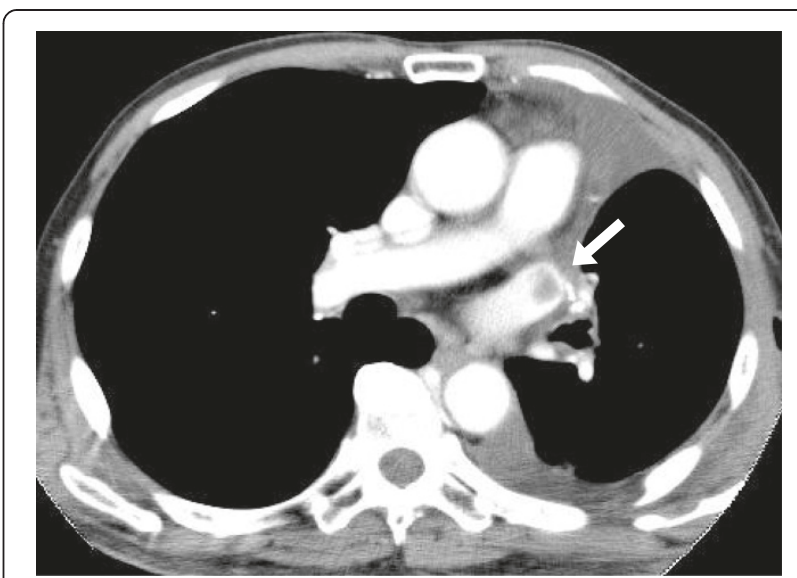

Figure $1 \mathrm{~A}$ thrombus in the left superior pulmonary vein stump after left upper lobectomy. Contrast-enhanced $C T$ on a 78 year-old male to check for other diseases on postoperative day 22 shows a thrombus in the LSPV stump.

The operative procedures consisted of 54 right upper lobectomies (RUL), 9 right middle lobectomies (RML), 30 right lower lobectomies (RLL), 28 LUL, and 30 left lower lobectomies (LLL). The operative approaches were 103 VATS, 14 hybrid VATS, and 34 open thoracotomies. The median operative time was 296 minutes (range: 143 to 482 minutes). The median amount of blood loss was $65 \mathrm{ml}$ (range: 0 to $1500 \mathrm{ml}$ ). The median postoperative hospital stay was 10 days (range: 5 to 179 days). Postoperative complications developed in 45 patients.

There were 139 cases of primary lung cancer, 10 of metastatic lung tumors, and 2 benign tumors. Of the primary lung cancers, there were 87 cases of adenocarcinoma, 35 of squamous cell carcinoma, 6 of adenosquamous cell carcinoma, 5 of small cell carcinoma, and 6 others. The median tumor size was $25 \mathrm{~mm}$ (range: 9 to

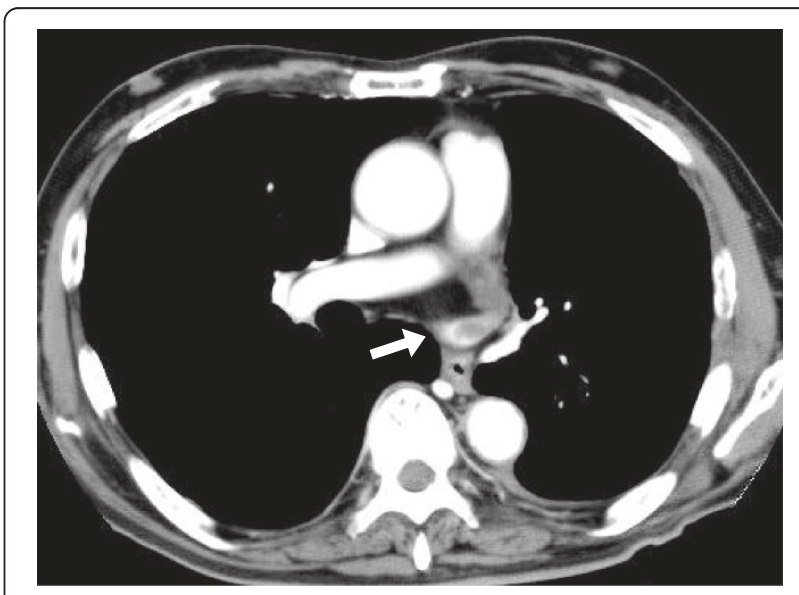

Figure $2 \mathrm{~A}$ thrombus in the left superior pulmonary vein stump after left upper lobectomy. Contrast-enhanced CT on a 66 year-old male for follow-up 7 months postoperatively shows a thrombus in the LSPV stump. This thrombus was not detected at that time. 
$67 \mathrm{~mm}$ ). Postoperative adjuvant chemotherapy was performed for 44 patients.

\section{Thrombosis in the PV stump after lobectomy}

Thrombosis in the PV stump after lobectomy developed in 5 of the 151 patients (3.3\%), all of whom had undergone LUL, and who accounted for 5 of the 28 patients who underwent that procedure (17.9\%). The thrombosis were found on contrast-enhanced CT for check-up of other disease within a month postoperatively in 2 patients and on contrast-enhanced CT for follow-up at 6 to 7 months postoperatively in 3 patients (Figures 1 and 2). These 5 patients did not have arrhythmia as atrial fibrillation and then infarction of any vital organ. In 2 patients, anticoagulant therapy was immediately started and $\mathrm{CT}$ a few months after the operation showed that the thrombus had disappeared. Then, anticoagulant therapy was stopped. In a patient, the thrombosis was not found at that time and disappeared after 9 months without any treatment. In 2 patients, the thrombosis is followed now with anticoagulant therapy.

\section{Risk factors for thrombosis in the PV stump after lobectomy} Clinicopathological factors were compared between the 5 patients with thrombosis in the PV stump and the 146 patients without thrombosis (Table 1). Only the operative

Table 1 Univariate analyses of clinicopathological factors associated with thrombosis in the pulmonary vein stump

\begin{tabular}{|c|c|c|c|}
\hline & $\begin{array}{l}\text { Patients with thrombosis } \\
\qquad(n=5)\end{array}$ & $\begin{array}{l}\text { Patients without thrombosis } \\
\qquad(\mathrm{n}=146)\end{array}$ & $P$ value \\
\hline Age, median value (range), years old & $75(56-81)$ & $72(38-82)$ & 0.5052 \\
\hline \multicolumn{4}{|l|}{ Sex, n (\%) } \\
\hline Male / Female & $4(80.0) / 1(20.0)$ & $97(66.4) / 49$ (33.6) & 0.4631 \\
\hline Brinkman index, median value (range) & $750(0-1200)$ & $600(0-3180)$ & 0.6092 \\
\hline \multicolumn{4}{|l|}{ Comorbidity, n (\%) } \\
\hline History of malignant disease & $2(40.0)$ & $37(25.3)$ & 0.3849 \\
\hline Hypertension & $2(40.0)$ & $77(52.7)$ & 0.4560 \\
\hline Diabetes mellitus & $0(0)$ & $22(15.1)$ & 0.4498 \\
\hline Cerebral infarction & $0(0)$ & $11(7.5)$ & 0.6815 \\
\hline Arrhythmia & $0(0)$ & $15(10.3)$ & 0.5883 \\
\hline Cardiovascular disease & $0(0)$ & $19(13.0)$ & 0.5055 \\
\hline Anti-coagulate drug & $0(0)$ & $38(26.0)$ & 0.2294 \\
\hline Steroids & $0(0)$ & $3(2.1)$ & 0.9033 \\
\hline \multicolumn{4}{|l|}{ Operative approach, n(\%) } \\
\hline Thoracotomy / VATS & $4(80.0) / 1(20.0)$ & $113(77.4) / 33(22.6)$ & 0.6858 \\
\hline \multicolumn{4}{|l|}{ Procedure, n (\%) } \\
\hline RUL, RML & $0(0)$ & $63(43.2)$ & $<0.001$ \\
\hline RLL & $0(0)$ & $30(20.5)$ & \\
\hline LUL & $5(100)$ & $23(15.8)$ & \\
\hline LLL & $0(0)$ & $30(20.5)$ & \\
\hline Operative time, median value (range), minutes & $301(240-425)$ & $295(143-482)$ & 0.8069 \\
\hline Dissection of mediastinal lymph nodes, n (\%) & $4(80.0)$ & $132(90.4)$ & 0.4117 \\
\hline Blood loss, median value (range), ml & $90(50-750)$ & $60(0-1500)$ & 0.2082 \\
\hline Duration of postoperative drainage, median value (range), days & $4(2-4)$ & $4(2-12)$ & 0.6149 \\
\hline Postoperative hospital stay, median value (range), days & $15(7-28)$ & $10(5-179)$ & 0.1828 \\
\hline Postoperative complication, n (\%) & $2(40.0)$ & $38(26.0)$ & 0.3990 \\
\hline \multicolumn{4}{|l|}{ Diagnosis, n (\%) } \\
\hline Primary / Metastasis / Benign & $5(100) / 0(0) / 0(0)$ & $134(91.8) / 10(6.8) / 2(1.4)$ & 0.7999 \\
\hline \multicolumn{4}{|l|}{ Pathological stage, n (\%) } \\
\hline | / || / ||| & $4(80) / 0(0) / 1(20)$ & $97(66.4) / 14$ (9.6) / 21 (14.4) & 0.7378 \\
\hline Adjuvant chemotherapy, n (\%) & $1(20.0)$ & $44(30.1)$ & 0.5310 \\
\hline
\end{tabular}


procedure showed a significant difference $(\mathrm{p}<0.001)$. Comorbidities, operative factors, and pathological factors did not show a significant difference.

\section{Discussion}

In this study, thrombosis in the PV stump developed in $3.3 \%$ of the patients who underwent lobectomy, and in $17.9 \%$ of those who underwent LUL. We previously reported that thrombosis developed in $3.6 \%$ of patients who underwent lobectomy and in $13.5 \%$ of those who underwent LUL [7]. Because the frequencies of thrombosis in the PV stump after lobectomy and after LUL were nearly equal in the two studies, this might be a common complication after LUL. Though this complication is not well known, it is very important because thrombosis in the systemic circulation might cause infarction of vital organs.

Clinically, thrombi in the systemic circulation typically develop as a result of atrial fibrillation. It is reported that left lobectomy might be a risk factor for atrial fibrillation [8]. However, of the 5 patients with thrombosis in this study, the 7 patients with thrombosis in our past study, and 6 patients in the past case report, none had atrial fibrillation when the thrombosis was diagnosed [2-7]. Moreover, all had undergone LUL, and none LLL. Therefore, the probability that atrial fibrillation or left lobectomy caused the thrombosis in the PV stump after lobectomy was low, whereas there was a high probability that LUL caused it.

In a previous study, we reported risk factors for thrombosis in the PV stump after lobectomy [7]. On univariate analysis comparing groups of patients with and without thrombosis in the PV stump, LUL and operative time showed significant differences, while postoperative adjuvant chemotherapy showed a marginal difference. In this study, there was a significant difference only for the operative procedure (Table 1). Based on our 2 studies in 2 institutes, LUL might be a risk factor for thrombosis in the PV stump after lobectomy.

We speculated that the cause of thrombosis in the left superior PV (LSPV) stump after LUL was the long LSPV stump. It might develop because turbulent flow or stasis of blood occurs in the long PV stump. Kwek et al. reported that thrombosis developed in longer PA stumps [9]. In the short PV stump, blood flow may occur because that in the left atrium spreads through the entire PV stump. In the long PV stump, turbulent flow or stasis of blood may occur because blood flow in the left atrium does not spread throughout the PV stump (Figure 3). In the right superior PV, because the branches to the upper lobe and middle lobe remain after RUL or RML, blood flow in the remaining branches spreads throughout the stump and turbulent flow or stasis of blood may not occur. In a previous report, we compared the lengths of

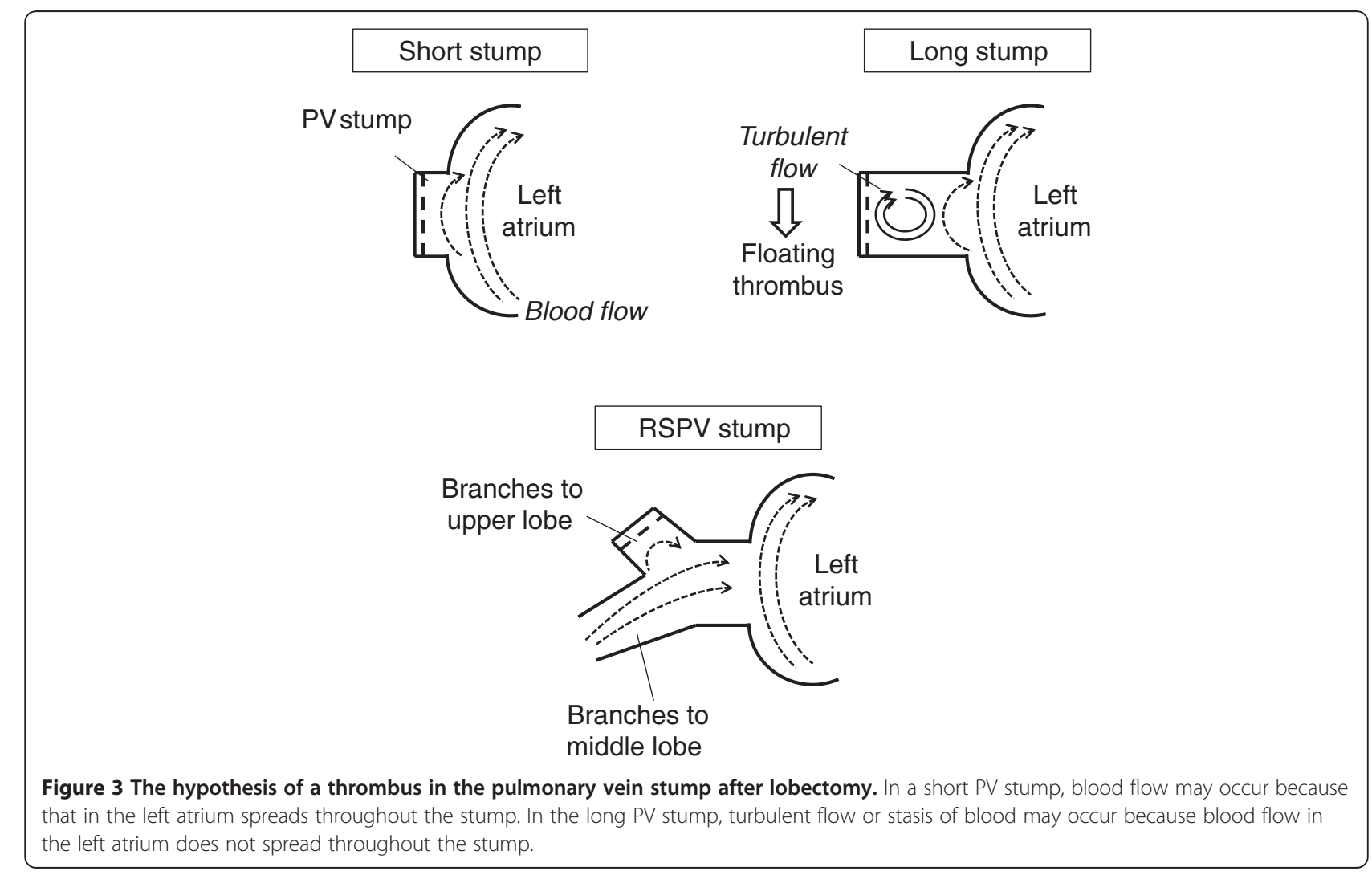


4. PV stumps after lobectomy using three-dimensional CT images [7]. As a result, it was demonstrated that the LSPV stump remained significantly longer than the other 3 PV stumps. Intrapericardial LSPV may be anatomically longer than the other $3 \mathrm{PVs}$. However, it has not yet been clarified whether turbulent flow or stasis of blood occurs and causes thrombosis in the long LSPV stump.

Why have there so few reports on thrombosis in the PV stump after lobectomy? We speculate that the reason may be that many institutes perform postoperative follow-up using chest X-rays or plane CT. Most guidelines recommend follow-up with plane $\mathrm{CT}$ or without $\mathrm{CT}$ after lung surgery [10]. If patients who undergo lobectomy are followed using contrast-enhanced CT and doctors conducting follow-up observe the PV stump on contrast-enhanced CT images, more patients with thrombosis in the PV stump may be detected.

At this time, we would propose the following measures with regard to thrombosis in the PV stump after lobectomy. First, the LSPV stump should be as short as possible. For example, after the LSPV is divided using a linear stapler, a ligation can be added to the LSPV stump near the pericardium to shorten it. Second, for early detection of possible thrombosis in the LSPV, contrast-enhanced CT must be performed as early as possible at least one time after LUL. Because it is difficult to demonstrate the PV stump by echocardiography after lobectomy, we recommend contrast-enhanced CT. Transesophageal echocardiography is useful, too. However transesophageal echocardiography may be painful test and more invasive than enhanced-CT because transesophageal echocardiography sometimes has a need for sedation. Third, if a thrombus in the LSPV stump is detected without an embolism, anticoagulant therapy should immediately be started. It seems to be premature to give thrombolytics because the benefit from it may not be greater than the risk of complications from it.

There are many questions regarding thrombosis of the PV stump. How often does this thrombosis generally occur? How often does this thrombosis cause infarction of vital organs? Is the risk of infarction of vital organs caused by this thrombosis higher than the risk of hemorrhagic event caused by anticoagulant therapy? Because there have been few reports about thrombosis in the LSPV stump after LUL, there is a pressing need for study in many institutes to clarify the frequency and risk factors for this complication. It is also an important issue that how aggressively the PV thrombi should be treated to prevent arterial infarctions.

\section{Conclusions}

In this study, it was clarified that the thrombosis in the LSPV stump after LUL was a common complication that potentially results in arterial thromboembolism.

\section{Abbreviations}

PV: Pulmonary vein; LUL: Left upper lobectomy; NSCLC: Non-small cell lung cancer; VATS: Video-assisted thoracoscopic surgery; PA: Pulmonary vein; RUL: Right upper lobectomy; RML: Right middle lobectomy; RLL: Right lower lobectomy; LLL: Left lower lobectomy; LSPV: Left superior pulmonary vein.

\section{Competing interests}

The authors declare that they have no competing interests.

\section{Authors' contributions}

$\mathrm{KO}$ performed the study design, the acquisition of data, and the statistical analysis, and drafted the manuscript. YH, KK, YT, HK, SH, TI, NS, NH, and YM helped to draft the manuscript. All authors read and approved the final manuscript.

\section{Author details}

'Department of Thoracic Surgery, Steel Memorial Muroran Hospital, 1-45 Chiribetsu-cho, Muroran, Hokkaido 050-0076, Japan. 'Department of Cardiovascular and Thoracic Surgery, Hokkaido University Graduate School of Medicine, Sapporo, Hokkaido, Japan.

Received: 20 August 2013 Accepted: 30 December 2013

Published: 6 January 2014

\section{References}

1. Ohtaka K, Hida Y, Kaga K, limura Y, Shiina N, Muto J, Hirano S: Pulmonary vein thrombosis after video-assisted thoracoscopic left upper lobectomy. J Thorac Cardiovasc Surg 2012, 143:e3-e5.

2. Nagaoka E, Yano M, Sugano T, Miyamoto T: Thrombus in the left superior pulmonary vein after left upper pulmonary lobectomy. J Thorac Cardiovasc Surg 2008, 135:709-710.

3. Schwalm S, Ward RP, Spencer KT: Transient ischemic attack in a patient with pulmonary vein thrombosis after left upper lobectomy for squamous cell lung cancer. J Am Soc Echocardiogr 2004, 17:487-488.

4. Seki M, Endo M, Kidani M, Kobayashi H, Sato H, Noto T: A rare case of left atrial thrombus after left upper pulmonary lobectomy [Article in Japanese]. Nippon Kyobu Geka Gakkai Zasshi 1989, 37:1371-1375.

5. Asteriou C, Barbetakis N, Efstathiou A, Kleontas A, Tsilikas C: Renal artery thrombosis following lobectomy for lung cancer. Case Rep Oncol 2010, 3:208-211.

6. Oura $\mathrm{H}$, Hirose $\mathrm{M}$, Aikawa $\mathrm{H}$, Ishiki M: Abdominal organ infarction encountered immediately after surgery of primary lung cancer [Article in Japanese]. Kyobu Geka 2005, 58:137-142.

7. Ohtaka K, Hida Y, Kaga K, Kato T, Muto J, Nakada-Kubota R, Sasaki T, Matsui $Y$ : Thrombosis in the pulmonary vein stump after left upper lobectomy as a possible cause of cerebral infarction. Ann Thorac Surg 2013, 95:1924-1928.

8. Xin Y, Hida Y, Kaga K, limura Y, Shiina N, Ohtaka K, Muto J, Kubota S, Matsui $Y$ : Left lobectomy might be a risk factor for atrial fibrillation following pulmonary lobectomy. Eur I Cardiovasc Thorac Surg 2013. in Press.

9. Kwek BH, Wittram C: Postpneumonectomy pulmonary artery stump thrombosis: CT features and imaging follow-up. Radiology 2005, 237:338-341.

10. Rubins J, Unger M, Colice GL: Follow-up and surveillance of the lung cancer patient following curative intent therapy. ACCP evidence-based clinical practice guideline (2nd Edition). Chest 2007, 132:355-367.

\section{doi:10.1186/1749-8090-9-5}

Cite this article as: Ohtaka et al.: Left upper lobectomy can be a risk factor for thrombosis in the pulmonary vein stump. Journal of Cardiothoracic Surgery 2014 9:5. 